\title{
MOMENTUM: momelotinib vs danazol in patients with myelofibrosis previously treated with JAKi who are symptomatic and anemic
}

\author{
Srdan Verstovsek ${ }^{1}$ (D) , Chih-Cheng Chen ${ }^{(\mathbb{D})}$, Miklós Egyed ${ }^{3}$, Martin Ellis $^{4}$, Laura Fox ${ }^{5}$ (D), \\ Yeow T Goh ${ }^{6}$, Vikas Gupta ${ }^{7}$ (D) Claire Harrison ${ }^{8}$, Jean-Jacques Kiladjian ${ }^{9}$ (D), Mihaela C \\ Lazaroiu $^{10}$, Adam Mead ${ }^{11}$ (D), Donal McLornan ${ }^{12}$, Mary F McMullin ${ }^{13}$ (i), Stephen T Oh ${ }^{14}$, \\ Andrew Perkins ${ }^{15}$ (D), Uwe Platzbecker ${ }^{16}$, Christof Scheid ${ }^{17}$, Alessandro Vannucchi ${ }^{18}$ (D), \\ Sung-Soo Yoon ${ }^{19}$, Mark M Kowalski*,20 \& Ruben A Mesa ${ }^{21}$ iD \\ ${ }^{1}$ Department of Leukemia at The University of Texas MD Anderson Cancer Center, Houston, TX 77030, USA \\ ${ }^{2}$ Division of Hematology, Chang Gung Memorial Hospital, Chiayi, Taiwan \\ ${ }^{3}$ Department of Hematology, Somogy County Mór Kaposi General Hospital, Kaposvár, 7400, Hungary \\ ${ }^{4}$ Hematology Institute \& Blood Bank, Meir Medical Center, Kfar Saba \& Sackler School of Medicine, Tel Aviv University, Tel \\ Aviv, 6997801, Israel \\ ${ }^{5}$ Department of Hematology, Vall d'Hebron Institute of Oncology (VHIO), Vall d'Hebron Hospital Universitari, Vall d'Hebron \\ Barcelona Hospital Campus, Barcelona, 08035, Spain \\ ${ }^{6}$ Singapore General Hospital, 169608, Singapore \\ ${ }^{7}$ Cancer Clinical Research Unit, Princess Margaret Cancer Centre, Toronto, ON, M5G 2C1, Canada \\ ${ }^{8}$ Guy's \& St. Thomas' NHS Foundation Trust, London, SE1 9RS, UK \\ ${ }^{9}$ Centre d'Investigations Cliniques (INSERM CIC 1427), AP-HP, Hopital Saint-Louis, Université de Paris, Paris, 75010, France \\ ${ }^{10}$ Policlinica de Diagnostic Rapid Brasov, 500152, Romania \\ ${ }^{11}$ Oxford University Hospitals NHS Foundation Trust, Oxford, OX3 9DU, UK \\ ${ }^{12}$ Guy's \& Saint Thomas' NHS Foundation Trust, London, UK \\ ${ }^{13}$ Queen's University Belfast, Belfast, BT9 7BL, Ireland \\ ${ }^{14}$ Hematology Division, Washington University, St. Louis, MO 63110, USA \\ ${ }^{15}$ The Alfred Hospital \& Monash University, Melbourne 3004, Australia \\ ${ }^{16}$ Leipzig University Hospital, Leipzig, 04103, Germany \\ ${ }^{17}$ Department of Internal Medicine \& Center of Integrated Oncology Cologne Bonn, University of Cologne, Köln, 50923, Germany \\ ${ }^{18}$ Center Research \& Innovation of Myeloproliferative Neoplasms, AOU Careggi, University of Florence, Firenze, 50139, Italy \\ ${ }^{19}$ Department of Internal Medicine, Seoul National University College of Medicine, Seoul National University Hospital, Seoul, Korea \\ ${ }^{20}$ Sierra Oncology, Vancouver, BC, V6C 3E8, Canada \\ ${ }^{21}$ Mays Cancer Center, UT Health San Antonio Cancer Center, San Antonio, TX 78229, USA \\ *Author for correspondence: mkowalski@sierraoncology.com
}

Hallmark features of myelofibrosis (MF) are cytopenias, constitutional symptoms and splenomegaly. Anemia and transfusion dependency are among the most important negative prognostic factors and are exacerbated by many JAK inhibitors (JAKi). Momelotinib (MMB) has been investigated in over 820 patients with MF and possesses a pharmacological and clinical profile differentiated from other JAKi by inhibition of JAK1, JAK2 and ACVR1. MMB is designed to address the complex drivers of iron-restricted anemia and chronic inflammation in MF and should improve constitutional symptoms and splenomegaly while maintaining or improving hemoglobin in JAKi-naive and previously JAKi-treated patients. The MOMENTUM Phase III study is designed to confirm and extend observations of safety and clinical activity of MMB.

Lay abstract: The most important features of myelofibrosis (MF) are low blood cell counts and symptoms including tiredness, night sweats and itching, along with increased size of the spleen, which may cause a feeling of fullness and pain. Low red blood cell counts (anemia) may mean regular blood transfusions are needed and this is one of the signs MF is getting worse. Drugs called JAK inhibitors (JAKi) are available to treat MF, but can have a side effect of making blood cell counts lower. Momelotinib (MMB) is a different type of JAKi to the ones currently available, and is an experimental drug for MF. MMB is designed to treat symptoms and spleen like other JAKi, but also to improve blood cell counts. MMB has already been given

Future Medicine 
to more than 820 patients with MF in other clinical studies. Some of the patients in these studies had been treated with different JAKi before, and others got MMB as their first JAKi treatment. The MOMENTUM Phase III study is designed to collect more information on the safety and effectiveness of MMB in MF.

First draft submitted: 15 October 2020; Accepted for publication: 9 December 2020; Published online: 11 January 2021

Keywords: ACVR1 • anemia • danazol • hemoglobin • JAK inhibitor • momelotinib • myelofibrosis • Phase III clinical trial • transfusion

Myelofibrosis (MF) is a chronic, progressive 'Philadelphia-negative' myeloproliferative neoplasm (MPN) with a prevalence of six per 100,000 person-years and a median age at diagnosis of 67 years [1,2]. MF may occur de novo as primary MF or may arise from a pre-existing MPN, primarily polycythemia vera or essential thrombocythemia [3]. The hallmark features of MF are: anemia and frequently transfusion dependency, often in association with thrombocytopenia; constitutional symptoms such as cachexia, fatigue, night sweats, fever and bone pain; and organomegaly due to extramedullary hematopoiesis, principally of the spleen and less often the liver, which can cause symptoms such as abdominal distension, pain and early satiety. Besides causing disease-related morbidity, MF may additionally result in premature death from complications including leukemic progression, which can occur in about $20 \%$ of patients, complications arising from progressive bone marrow failure, portal or pulmonary hypertension, infections, thrombosis, bleeding and cardiovascular complications $[4,5]$. The median survival for all patients with MF is approximately 6 years but is considerably worse for intermediate 2- and high-risk patients at 4 years and 2.25 years, respectively [5,6].

\section{Anemia}

The etiology of anemia in MF is complex and driven by multiple inter-related factors including hyperactivation of ACVR1 and dysregulated JAK-STAT signaling. Constitutively active JAK-STAT signaling leads to increased expression of inflammatory cytokines in the bone marrow resulting in progressive fibrosis, and a systemic proinflammatory cytokine profile that drives the production of the master iron regulator, hepcidin. Elevated levels of hepcidin sequester iron from the circulation, further reducing erythropoietic capacity and resulting in iron-restricted anemia. Anemia can be further exacerbated by the approved JAK inhibitors (JAKi) ruxolitinib (jakafi/jakavi) and fedratinib (inrebic), given that these agents are unable to restore iron homeostasis and effective erythropoiesis due to a lack of ACVR1 inhibitory activity [3].

Transfusion dependency and moderate to severe anemia are critical negative prognostic factors in MF and inversely correlated with quality of life (QoL) [7-9]. Severe anemia is associated with a greater than 1.5-fold increase in risk of death compared with moderate anemia and is associated with a median survival of just 2.1 years. Prognostic models for MF increasingly recognize anemia as an important risk variable $[10,11]$. The only viable treatment option for most transfusion-dependent patients with MF remains red blood cell (RBC) transfusions, despite this approach having no impact on the underlying disease [8]. Transfusions themselves are a substantial burden to the patient and healthcare systems, since QoL and overall survival in MF are negatively impacted, and complications relating to iron overload may arise [3,12,13].

\section{MF symptoms \& splenomegaly}

MF is a chronic disease characterized by a high symptom burden, reduced QoL, shortened survival and disease complications such as an anemia of chronic disease, immune dysregulation and leukemic progression. Patients responding to an online survey of MPN-related symptoms and the impact of MPNs on QoL and ability to work (MPN landmark [14]) reported that MF reduced their QoL (81\%), interfered with daily activities (53\%) and resulted in limits to their work productivity (65\% of employed respondents). Fatigue has been identified as the most common and most severe symptom of MF along with constitutional symptoms associated with systemic inflammation, abdominal symptoms secondary to splenomegaly and symptoms of anemia [14,15]. In addition to contributing to symptom burden, splenomegaly in MF can be particularly onerous and result in morbidities including ischemia, splenic infarction and development or exacerbation of cytopenias. JAKi therapies have been shown to provide both symptomatic and splenic benefit to patients with MF. 


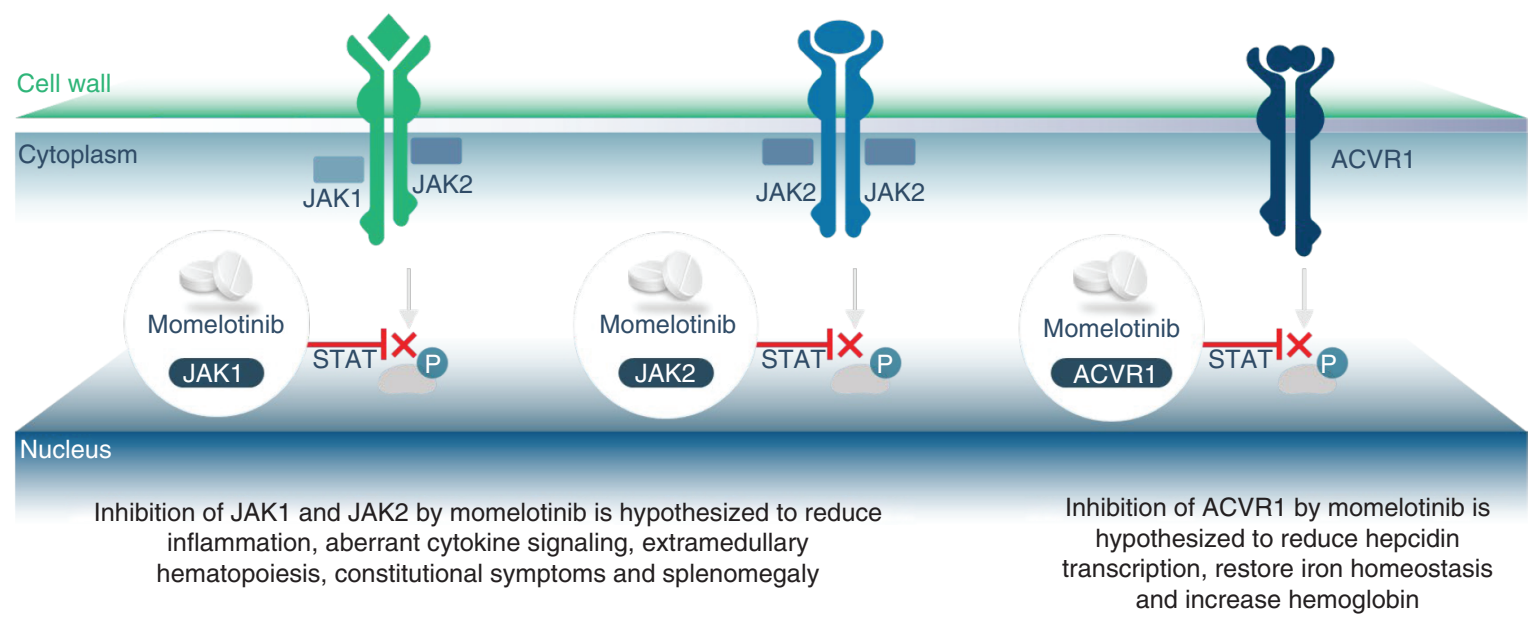

Figure 1. Momelotinib therapy has the potential to decrease inflammation, improve splenomegaly and normalize hemoglobin.

\section{Current therapies}

Allogeneic hematopoietic stem cell transplantation is the only curative treatment for MF and is particularly challenging for patients with the condition because of their advanced age, frequent competing co-morbidities and potential lack of viable donor options. This procedure requires a careful risk/benefit assessment as there is a high risk of transplant-related mortality and 5-year survival rates are only 37 and $22 \%$ in high- and very high-risk patients (83 and 64\% in low- and intermediate-risk patients) [16].

Before the availability of JAKi, therapy for MF was mainly palliative and directed toward amelioration of disease sequelae and symptom management. The discovery of the JAK2 driver mutation in MPNs led to the development of several JAKi. Small-molecule inhibitors of JAK2 and/or JAK1 have been developed to inhibit pathogenic JAK-STAT signaling in MPNs and have demonstrated spleen and symptom benefit in patients with MF with or without the JAK2 V617F mutation, consistent with a demonstrated ability to inhibit both mutant (V617F) and wild-type JAK2 signaling $[17,18]$. The currently marketed JAKi ruxolitinib (RUX) and fedratinib provide symptomatic and splenic benefit in patients with MF, and as such are valuable additions to MF therapy. However, these agents frequently exacerbate cytopenias which may limit adequate dose intensity. Consequently, anemia and thrombocytopenia have remained significant challenges in the management of MF [3].

Existing approaches for the management of MF-associated anemia include transfusion, erythropoiesis-stimulating agents in patients with low serum erythropoietin levels, corticosteroids, androgens (including danazol [DAN]), immunomodulatory imide drugs and splenectomy. The clinical activity of these has been modest and often limited to patient subsets, and none improve survival $[3,19-21]$. This minimal effectiveness may be attributed to their inability to directly address the underlying causes of MF-associated anemia, including chronic inflammation and iron restriction within erythroid progenitors [22-25].

\section{Momelotinib}

MMB is an investigational agent in development for the treatment of intermediate and high-risk MF. It is a potent oral inhibitor of JAK1, JAK2 and, uniquely among JAKi in development, an inhibitor of ACVR1 (Figure 1). Inhibition of JAK1 and JAK2 signaling reduces constitutional symptoms and splenomegaly through decreased aberrant inflammatory cytokine signaling, mutant hematopoietic stem cell proliferation and RBC sequestration. In addition, MMB's inhibition of ACVR1 decreases hepcidin [26,27], the master regulator of iron metabolism that is elevated in patients with MF. Decreasing hepcidin results in restoration of iron homeostasis leading to increased serum iron availability for erythropoiesis, facilitating normalized hemoglobin levels and decreased RBC transfusion requirements.

Prior to the initiation of the current Phase III MOMENTUM study, MMB has been studied in a comprehensive clinical development program with more than 20 Phase I, II and III clinical studies enrolling in aggregate more than 1220 subjects, including more than 820 patients with MF. 
SIMPLIFY-1 (S1) was a Phase III, 1:1 randomized, double-blind, head-to-head, noninferiority comparison of MMB to RUX in JAKi-naive subjects with MF ( $\mathrm{n}=432)$. The study had a duration of approximately 5.4 years. MMB demonstrated a statistically noninferior splenic response rate (SRR) to RUX (27\% for MMB vs $29 \%$ for RUX) and the transfusion independence (TI) rate at week 24 was higher in the MMB arm (67\% for MMB vs $49 \%$ for RUX [p $<0.001])$. Based on the predefined Myelofibrosis Symptom Assessment Form Total Symptom Score (MFSAF TSS) end point, a 50\% or greater reduction in the TSS was observed in 28 and $42 \%$ of patients who received MMB and RUX, respectively, indicating that noninferiority was not met ( $\mathrm{p}=0.97)$. The MOMENTUM study is stratified for baseline TSS, in contrast to S1 where an imbalance in baseline TSS levels may have confounded the outcome. During randomized treatment, the most common grade 3 or 4 treatment-emergent adverse events were thrombocytopenia occurring in 7\% of patients receiving MMB versus 5\% for RUX, and anemia occurring in $6 \%$ for MMB versus $23 \%$ for RUX. Grade 3 or 4 infections occurred in $7 \%$ of patients who received MMB and $3 \%$ of patients who received RUX. Treatment-emergent peripheral neuropathy occurred in $10 \%$ of patients who received MMB (all of grade 2 or lower) and 6\% of patients who received RUX (all of grade 3 or lower).

SIMPLIFY-2 (S2) was a Phase III, 2:1 randomized study comparing MMB to best available therapy (BAT), which was approximately 90\% RUX, in anemic or thrombocytopenic subjects with MF previously treated with RUX ( $\mathrm{n}=156)$. The study had a duration of approximately 4.8 years. Statistical superiority was not demonstrated for spleen response, the primary end point. However, it is important to note that a washout from prior RUX therapy was prohibited per protocol, which may have confounded the baseline spleen assessment. To mitigate against this potentially confounding effect, the MOMENTUM study requires the absence of active anti-MF therapy (including JAKi) for 2 or more weeks prior to study randomization, with the baseline spleen size assessment obtained as close to randomization as feasible. In the analysis of the secondary end points in S2, MMB demonstrated a nominally statistically superior MFSAF TSS response to BAT (26\% for MMB vs $6 \%$ for BAT [p = 0.001]) and the TI rate at week 24 was nominally higher in the MMB arm ( $43 \%$ for MMB vs $21 \%$ for BAT [p = 0.001]). The SRR at the end of randomized treatment (spleen volume decreased by at least 35\% compared with baseline) was $7 \%$ for the MMB arm versus $6 \%$ for BAT $(\mathrm{p}=0.90)$. During randomized treatment, the most common grade 3 or 4 treatmentemergent adverse events were anemia occurring in 13\% for MMB versus 17\% for BAT, and thrombocytopenia occurring in $11 \%$ of patients receiving MMB versus $6 \%$ for BAT. Peripheral neuropathy occurred in $12 \%$ of patients receiving $\mathrm{MMB}$ and in no patients in the BAT group.

Long-term clinical data for MMB are available from S1, S2 and an extended access protocol (XAP) enrolling 137 patients following the completion of S1, S2 and earlier Phase II MMB studies. A total of 105 subjects remain on XAP, some of whom have received MMB for more than 10 years as of September 2020. Analyses of data from S1, S2 and XAP demonstrate low rates of high-grade hematological and other toxicities. Safety observations were generally similar for MMB versus RUX during double-blind treatment in S1 and S2, though notably anemia and thrombocytopenia were more common in the RUX arm of S1 and more dose limiting, while low-grade nausea was more common in the MMB arms of both S1 and S2. Tolerability of extended treatment has enabled long duration dosing with no evidence of long-term cumulative toxicity over 3.5 to 5.5 years of follow-up and more than 90 patients from S1 and S2 continuing to receive MMB. Also in S1 and S2, reduced transfusion burden and increased dose intensity have been observed compared with RUX, consistent with the compound's differentiated pharmacological and clinical profile [28-30].

\section{Introduction to the MOMENTUM trial}

Here, we describe MOMENTUM (SRA-MMB-301) 'a randomized, double-blind, phase iii study to evaluate the activity of momelotinib versus danazol in symptomatic, anemic subjects with primary myelofibrosis, postpolycythemia vera myelofibrosis, or post-essential thrombocythemia myelofibrosis who were previously treated with jak inhibitor therapy' (ClinicalTrials.gov: NCT04173494). The study sponsor is Sierra Oncology, Inc. headquartered in Vancouver, Canada.

\section{Rationale}

MOMENTUM is a global, Phase III study designed to confirm and extend preliminary observations of safety and of the symptomatic, splenic and anemia benefits of MMB made in the prior Phase III SIMPLIFY studies. Eligible patients represent a population with an unmet medical need, having previously received treatment with an approved JAKi and being both symptomatic and anemic at baseline. 


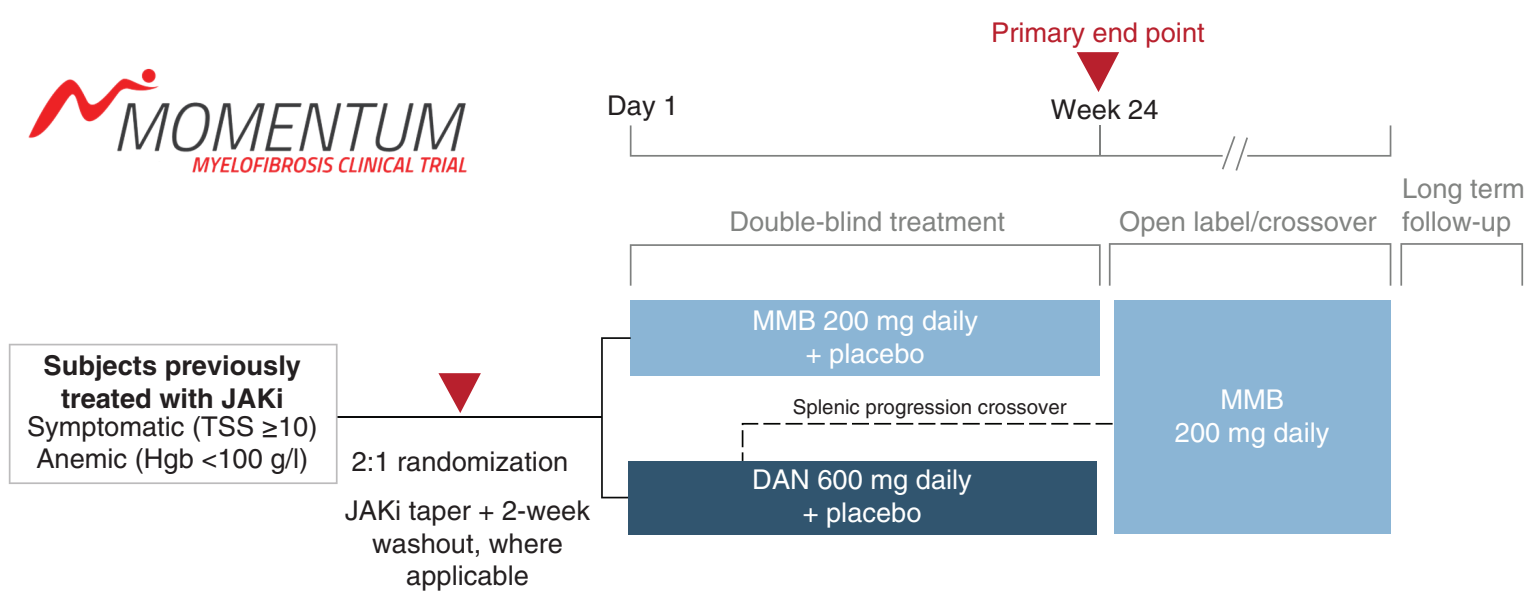

Figure 2. MOMENTUM study schematic.

DAN: Danazol; JAKi: JAK inhibitor; MMB: Momelotinib; TSS: Total symptom score.

Improvement in symptoms assessed by the MFSAF TSS version 4.0 has been selected as the primary end point. The MFSAF is a validated patient-reported outcome (PRO) instrument, which measures the core symptoms of MF [31]. This instrument assesses the therapeutic benefit of MMB on symptom burden, a cardinal feature of MF.

There are no approved therapies for MF specifically labeled for use in patients previously treated with a JAKi, however, guidelines do recommend a number of treatment options. With consideration to potential risk and benefit to patients and how readily a comparator treatment could be used in a double-blind study, DAN, a semisynthetic-attenuated androgen, was selected as an appropriate treatment comparator given its use to ameliorate anemia in patients with advanced MF and recommendation by National Comprehensive Cancer Network and European Society for Medical Oncology guidelines for the management of MF-associated anemia [22,32]. Treatment with DAN elicits similar benefit to other anabolic steroids used in the treatment of anemia with less toxicity [33].

\section{Design}

MOMENTUM is a randomized, double-blind Phase III study of $200 \mathrm{mg}$ MMB daily versus $600 \mathrm{mg}$ DAN daily $(2: 1)$ in previously JAKi-treated patients who are symptomatic and anemic. Eligible patients have previously received approved JAKi therapy for MF for a minimum of 90 days, or a minimum of 28 days if JAKi therapy was complicated by RBC transfusion requirement of at least four units in 8 weeks, or a Grade 3/4 adverse events (AEs) of thrombocytopenia, anemia, or hematoma. Patients must be symptomatic with an MFSAF TSS of 10 or greater at screening, and anemic with hemoglobin less than $100 \mathrm{~g} / \mathrm{l}$ (Table 1). Enrollment of approximately 180 subjects is planned (Figure 2). The primary end point of the study is the MFSAF TSS response rate at week 24 and key secondary end points include the TI rate at week 24 and SRR at week 24 (Table 2).

In order to more accurately assess the comparative efficacy of MMB and DAN, any ongoing JAKi therapy must be tapered, followed by a 2-week nontreatment interval prior to initiating study treatment. Subjects are randomized on a 2:1 basis (MMB plus DAN placebo: DAN plus MMB placebo), stratified by baseline MFSAF TSS, baseline palpable spleen length and baseline RBC units transfused in the 8-week period prior to randomization. In order to minimize delay between baseline assessments and randomization, palpable spleen length will be used for stratification; however, spleen volume measured by CT or MRI will be used for assessment of splenic response. During the randomized treatment period of 24 weeks, subjects orally self-administer blinded, randomized treatment. Treatment may be interrupted and/or dose reduced due to thrombocytopenia, neutropenia, nonhematologic or other toxicities according to protocol-specified criteria.

Assessments for the primary and key secondary end points of the study (Table 2) are performed during randomized treatment; the MFSAF TSS version 4.0 questionnaire will be completed daily and transfusion/CBC recording every 4 weeks. A spleen volume assessment (for assessment of splenic response) is conducted at the end of randomized treatment at week 24 and also at week 48 during open-label treatment. Other assessments during this period 


\section{Table 1. Key eligibility criteria for MOMENTUM.}

\section{Key inclusion criteria}

\section{- Age $\geq 18$ years}

- Confirmed diagnosis of PMF in accordance with the WHO 2016 criteria, or post-PV/ET MF in accordance with the IWG-MRT criteria

- Symptomatic, defined as an MFSAF TSS of $\geq 10$ units assessed by a single MFSAF

v4.0 assessment

- Anemic, defined as hemoglobin $<100 \mathrm{~g} / \mathrm{l}$

- Previously treated, with an approved JAKi for PMF or post-PV/ET MF for $\geq 90$ days, or $\geq 28$ days if JAKi therapy is complicated by RBC transfusion requirement of $\geq 4$ units in 8 weeks, or grade $3 / 4 \mathrm{AEs}$ of thrombocytopenia, anemia or hematoma

- Ongoing JAKi therapy must be tapered, and a nontreatment interval beginning 7 days prior to the first of seven consecutive days of baseline MFSAF assessments

- Baseline splenomegaly, defined as having a palpable spleen at $\geq 5 \mathrm{~cm}$, below the

LCM or with volume $\geq 450 \mathrm{~cm}^{3}$ on imaging

- High risk, intermediate-2 or intermediate-1 risk as defined by DIPSS or DIPSS-plus

- No allogeneic stem cell transplant planned

- Acceptable laboratory assessments:

- ANC $\geq 0.75 \times 10^{9} / 1$

- PLT $\geq 25 \times 10^{9} / 1$

- Peripheral blast count $<10 \%$

- AST/SGOT and ALT/SGPT $\leq 3 \times$ ULN ( $\leq 5 \times$ ULN if liver is involved by

extramedullary hematopoiesis as judged by the investigator or if related to iron

chelator therapy that was started within the prior 60 days)

$-\mathrm{CrCl} \geq 0.5 \mathrm{ml} / \mathrm{s}$ (according to Cockcroft-Gault)

- Direct bilirubin $\leq 2.0 \times$ ULN

- ECOG performance status of 0,1 or 2

- Life expectancy $>24$ weeks
Key exclusion criteria

- Use of the following treatments within the time periods noted prior to initiating study treatment:

- MMB at any time

- DAN within 3 months

- JAKi therapy within 2 weeks

- ESA within 4 weeks

- Potent CYP450 3A4 (CYP3A4) inducers within 1 week

- Investigational agent within 4 weeks

- Splenic irradiation within 3 months

- Other active anti-MF therapy within 2 weeks including: alkylating agents, hypomethylating agents, interferons, immunomodulating agents, corticosteroids, androgens, growth factors, splenic irradiation, splenectomy. Supportive care including steroids at a dose equivalent to $\leq 10 \mathrm{mg}$ prednisone per day for non-MF indications may be used

- Current treatment with simvastatin, atorvastatin, lovastatin or rosuvastatin - History of prostate cancer, with the exception of localized prostate cancer that has been treated surgically or by radiotherapy with curative intent and presumed cured - PSA $>4 \mu \mathrm{g} / \mathrm{l}$

- Unsuitable for spleen volume measurements

- Any of the following within the time periods noted prior to initiating study treatment:

- Current uncontrolled intercurrent illness including, but not limited to: active uncontrolled infection

- Significant active or chronic bleeding event $\geq$ grade 2 per CTCAE v5.0, within 4 weeks

- Unstable angina pectoris, symptomatic congestive heart failure or uncontrolled cardiac arrhythmia within 6 months

- Current QTcF interval $>500$ ms, unless attributed to bundle branch block

- Current progressive thrombosis despite treatment

- History of porphyria

- Current Child-Pugh score $>10$

- Known clinically significant anemia due to iron, vitamin B12 or folate deficiencies, or autoimmune or hereditary hemolytic anemia, or gastrointestinal bleeding

- Known positive status for HIV

- Chronic active or acute viral hepatitis A, B or C infection, or hepatitis B or C carrier

- Unresolved nonhematologic toxicities from prior therapies that are $>$ grade 1 per

CTCAE v5.0

- Presence of peripheral neuropathy $\geq$ grade 2 per CTCAE v5.0

AE: Adverse event: ANC: Absolute neutrophil count: $\mathrm{CrCl}$ : Calculated creatinine clearance: CTCAE: Common Terminology Criteria for Adverse Events; DAN: Danazol: ECOG: Eastern Cooperative Oncology Group; ESA: Erythropoiesis-stimulating agent; ET: Essential thrombocythemia; ICF: Informed consent form; IWG-MRT: International Working Group-Myeloproliferative Neoplasms Research and Treatment; JAKi: JAK inhibitor; LCM: Left costal margin; MFSAF TSS: Myelofibrosis symptom assessment form total symptom score; MMB: Momelotinib; PLT: Platelet count; PMF: Primary myelofibrosis; PRO: Patient-reported outcome; PSA: Prostate-specific antigen; PV: Polycythemia vera; RBC: Red blood cell count; ULN: Upper limit of normal; WOCBP: Women of childbearing potential.

\section{Table 2. Key end points.}

End poin

Primary end point

Key secondary end points

Key exploratory end points
Assessment

The MFSAF TSS response rate at week 24; defined as the proportion of subjects who achieve $a \geq 50 \%$ reduction in TSS over the 28 days immediately prior to the end of week 24 compared with baseline

- Proportion of subjects with TI status at the end of week 24 defined as not requiring $\mathrm{RBC}$ transfusion (except in the case of clinically overt bleeding) for $\geq 12$ weeks immediately prior to the end of week 24 , with hemoglobin level $\geq 80 \mathrm{~g} / \mathrm{l}$. Assessed in all subject

- sSRR defined as the proportion of subjects who have splenic response (reduction in spleen volume of $\geq 35 \%$ from baseline) at the end of week 24

- Other secondary end points include mean change from baseline MFSAF

TSS, measures of anemia benefit and duration of response, safety assessments, survival analyses, change from baseline in PROs and plasma concentration of MMB

Exploratory end points include measures of rate and duration of MFSAF TSS response, time to splenic progression, correlated of response and exploratory analysis (including mutational analysis) and health resource utilization

MFSAF TSS: Myelofibrosis Symptom Assessment Form v4.0 Total Symptom Score; MMB: Momelotinib; PRO: Patient-reported outcome; RBC: Red blood cell count; SRR: Splenic response rate; TI: Transfusion independence. 
include PRO questionnaires to assess fatigue, physical function and overall QoL, also safety assessments including clinical, laboratory and disease assessments, recording of AEs, concomitant medications, laboratory tests (chemistry, complete blood count, urinalysis, pregnancy, electrocardiogram), physical examinations and vital signs.

Following the completion of all week 24 assessments, subjects have the option to receive MMB in the open-label extended treatment period. Early crossover to open-label MMB is also available for subjects who meet the protocoldefined criteria for radiographically confirmed splenic progression. Open-label treatment with MMB may continue up to the end of week 204, dependent on jurisdiction. During open-label treatment, MFSAF TSS assessments are performed at 4-week intervals until week 48, along with continued recording of RBC transfusions, PROs and safety assessments. Spleen volume is assessed at week 48 and also as required to confirm splenic progression. Additional assessments include gathering of health resource utilization data, pharmacokinetic assessments and exploratory mutational analysis. Leukemia-free survival and overall survival is assessed during a follow-up period, which may extend the maximum study participation to approximately 7 years.

During the conduct of the trial, a Data Monitoring Committee will review the progress of the clinical trial, safety data, critical efficacy end points and make recommendations to the sponsor regarding the continued conduct of the study.

\section{Statistical methods}

Three clinically relevant measures of the subject response are being investigated in this study: symptom burden, TI and spleen response. Sequential testing will be used to control Type I error. All analyses will employ the subject stratification based on baseline TSS, baseline number of transfusions and baseline spleen size.

The primary end point of TSS response rate at week 24 will be a test for superiority of MMB relative to DAN. The TI rate will be tested for noninferiority of MMB relative to DAN and then for superiority if noninferiority is obtained. The SRR will be tested for superiority.

Additional secondary end points for symptom response include the difference in mean change TSS from baseline to the end of week 24 using a repeated measures model and the duration of the week 24 TSS response assessed to the end of week 48 .

The planned 180 subjects in this study provide power to detect with a two-sided $\alpha$ of 0.05 and randomization ratio 2:1 for MMB:DAN, clinically relevant, statistically significant improvements in TSS response rate $(98.8 \%$ power), TI status (90\% power) and SRR (90\% power).

\section{Conclusion}

MMB possesses a pharmacological and clinical profile differentiated from other JAKi in development by virtue of its potent inhibition of JAK1, JAK2 and ACVR1. Consequently, MMB may address the complex drivers of iron-restricted anemia and chronic inflammation in MF and should improve constitutional symptoms and splenomegaly while maintaining or improving hemoglobin across the continuum of JAKi-naive and previously JAKi-treated patients.

More than 820 patients with MF have received MMB, including 555 patients enrolled in the two Phase III SIMPLIFY studies. Following participation in the SIMPLIFY studies and earlier Phase II studies of MMB, patients with intermediate-/high-risk MF have received extended MMB treatment ranging to more than 10 years duration. Analyses of long-term clinical data revealed no new safety signals or cumulative toxicity with extended treatment, demonstrating MMB's potential to durably address the unmet needs of patients with MF.

MMB remains an investigational agent under development in MOMENTUM, a global Phase III study designed to support the preliminary safety findings and activity observed across the prior Phase III SIMPLIFY studies of MMB evidenced by responses in symptoms, spleen and anemia end points. In addition to these metrics, MOMENTUM will provide an opportunity to further evaluate associations between anemia benefit, transfusion burden and patient reported measures of clinical benefit. 
Executive summary

Background \& rationale

- Momelotinib (MMB), an investigational therapy for myelofibrosis (MF), possesses a pharmacological and clinical profile differentiated from other JAK inhibitors (JAKi) by virtue of its potent inhibition of JAK1, JAK2 and ACVR1. Consequently, MMB addresses the complex drivers of iron-restricted anemia and chronic inflammation in MF and should improve constitutional symptoms and splenomegaly while maintaining or improving hemoglobin across the continuum of JAKi-naive and previously JAKi-treated patients.

- More than 820 patients with MF have received MMB, including in two Phase III studies. Analysis of clinical data demonstrate MMB's potential to address the unmet needs of patients with intermediate-/high-risk MF through an absence of significant rates of high-grade hematological and other toxicities, reduced transfusion burden and increased dose intensity.

- The comparator treatment, danazol (DAN), is recommended by treatment guidelines for amelioration of anemia in patients with advanced MF.

MOMENTUM study design \& key eligibility criteria

- MOMENTUM is a randomized (2:1, MMB:DAN), double-blind study in approximately 180 patients who are symptomatic and anemic having received prior JAKi therapy.

- Eligible patients will have previously received approved JAKi therapy for MF for a minimum of 90 days, or a minimum of 28 days if JAKi therapy was complicated by red blood cell transfusion requirement of $\geq 4$ units in 8 weeks, or grade $3 / 4$ adverse events of thrombocytopenia, anemia or hematoma.

- Patients must be symptomatic with a Myelofibrosis Symptom Assessment Form Total Symptom Score (MFSAF TSS) of $\geq 10$ at Screening, and anemic with hemoglobin $<100 \mathrm{~g} / \mathrm{l}$.

Outcome measures

- The primary end point is the MFSAF TSS response rate (defined as the proportion of subjects who achieve $a \geq 50 \%$ reduction in TSS over 28 days) at week 24.

- Key secondary end points include the proportions of subjects with transfusion independent status at week 24 and splenic response rate at week 24 .

- Other secondary end points include mean change from baseline MFSAF TSS, measures of anemia benefit and duration of response, safety assessments, survival analyses, change from baseline in patient-reported outcomes and plasma concentration of MMB.

Conclusion

- MOMENTUM is a global, Phase III study designed to confirm and extend preliminary observations of safety and of the symptomatic, splenic and anemia benefits of MMB made in the prior Phase III SIMPLIFY studies.

Financial \& competing interests disclosure

The study was sponsored by Sierra Oncology, Inc. The authors C-C Chen, M Egyed, M Ellis, YT Goh, MC Lazaroiu and C Scheid have no potential conflict of interest to declare. S Verstovsek received honoraria and research funding from Sierra Oncology and research funding from Incyte Corporation, Roche, NS Pharma, Celgene, Gilead, Promedior, CTI BioPharma Corp., Genentech, Blueprint Medicines Corp., Novartis, Pharma Essentia, AstraZeneca, Ital Pharma, Protagonist Therapeutics, Constellation Pharmaceuticals, Kartos Therapeutics, Prelude Therapeutics, AbbVie, Inc., Telios Pharmaceuticals and Galecto. L Fox participated in advisory boards for Novartis and served in a volunteer capacity as a member of the Gemfin board. V Gupta received honoraria from Sierra Oncology, Celgene and Pfizer, and research funding plus honoraria from Novartis. C Harrison was a consultant for Galacteo, Sierra Oncology, Constellation, Promeditor and Geron; was a consultant, received honoraria and participates in speaker's bureau for Celgene and Novartis; was a consultant and participated in speaker's bureau for CTI; was a consultant for and received honoraria from Roche; received honoraria from AOP Pharma; participated in speaker's bureau for Jannsen. J-J Kiladjian received research funding and participates in advisory boards for Novartis and AOP Orphan. A Mead was a consultant for AbbVie; is a consultant, received honoraria and research funding and participated in speaker's bureau for Novartis; was a consultant and received research funding from Celgene and BMS; received research funding and honoraria from CTI. D McLornan received travel bursary and speaker fees and participated in advisory boards for Jazz Pharmaceuticals; received speaker fees and participated in advisory boards for Novartis; and participated in advisory boards for Celgene/BMS. MF McMullin participated in advisory Boards for Daiiyo Sanyo, Astellas, Celgene and AbbVie; received speaker fees from Novartis and Jazz Pharmaceuticals. ST Oh was a consultant and participated in advisory boards for Novartis, Kartos Therapeutics, CTI BioPharma, Celgene/Bristol Myers Squibb, Disc Medicine, Blueprint Medicines, PharmaEssentia, Constellation and Incyte. A Perkins received honoraria Novartis and participates in MPN Advisory Boards for Novartis and Kartos Therapeutics. U Platzbecker was a consultant and received research funding from Celgene and Novartis, and received research funding from Amgen, Janssen Biotech and Merck. A Vannucchi participated in speaker's bureau and had membership on the Board of Directors or advisory committees for Novartis, BMS/Celgene and AbbVie; membership on the Board of 
Directors or advisory committees for Blueprint and Incyte. S-S Yoon had a consulting or advisory role for Takeda, Amgen, Astellas, Celgene, Chugai, Janssen, Novartis; received honoraria from Novartis and Amgen; received research funding from Kyowa Kirin, Roche-Genentech and Yuhan Pharmaceutical. M Kowalski was employed by and held stock in Sierra Oncology. RA Mesa was a consultant for Novartis, Sierra Oncology and LaJolla, and received research support from Celgene, Incyte, AbbVie, Samus, Genetech, Promedior and CTI. The authors have no other relevant affiliations or financial involvement with any organization or entity with a financial interest in or financial conflict with the subject matter or materials discussed in the manuscript apart from those disclosed. Andrew Dye, an employee of Sierra Oncology, provided medical writing support for the manuscript.

Ethical conduct of research

The authors state that they have obtained institutional review board approval from all investigational sites for the research described. In addition, they have obtained verbal and written informed consent from the patients for the inclusion of their medical and treatment history within this work.

\section{Data sharing statement}

Sierra Oncology Inc. supports the principles of clinical trial data transparency, including registration and disclosure of clinical trial results in external registries and publication of results in peer-reviewed journals. Individual deidentified participant data (including data dictionaries) will not be shared; however, initial data will be shared publicly via press-release following analysis of all subject's data from the randomized period of the trial. Results of the study will be made available indefinitely via Clinicaltrials.gov and other registries within 12 months of completion of the trial. Results will also be presented at appropriate conference(s) and via journal publication. Data and other documents such as the study protocol and statistical analysis plan may also be made available, as needed, to researchers who provide a methodologically sound proposal.

\section{Open access}

This work is licensed under the Attribution-NonCommercial-NoDerivatives 4.0 Unported License. To view a copy of this license, visit http://creativecommons.org/licenses/by-nc-nd/4.0/

\section{References}

Papers of special note have been highlighted as: $\bullet$ of interest; $\bullet \bullet$ of considerable interest

1. O'Sullivan JM, Harrison CN. Myelofibrosis: clinicopathologic features, prognosis, and management. Clin. Adv. Hematol. Oncol. 16(2), 121-131 (2018).

2. Iurlo A, Cattaneo D. Treatment of myelofibrosis: old and new strategies. Clin. Med. Insights Blood Disord. 10, 1-10 (2017).

3. Naymagon L, Mascarenhas J. Myelofibrosis-related anemia: current and emerging therapeutic strategies. HemaSphere 1(1), e1 (2017).

-. This review describes how anemia and thrombocytopenia have remained challenges in the management of myelofibrosis (MF) despite the availability of ruxolitinib.

4. Vainchenker W, Leroy E, Gilles L, Marty C, Plo I, Constantinescu SN. JAK inhibitors for the treatment of myeloproliferative neoplasms and other disorders. F1000 Res. 7(0), 82 (2018).

5. Zahr AA, Salama ME, Carreau N et al. Bone marrow fibrosis in myelofibrosis: pathogenesis, prognosis and targeted strategies. Haematologica 101(6), 660-671 (2016).

6. Cervantes F, Dupriez B, Pereira A et al. New prognostic scoring system for primary myelofibrosis based on a study of the International Working Group for Myelofibrosis Research and Treatment. Clin. Trials Obs. 113(13), 2895-2901 (2009).

7. Tefferi A, Lasho TL, Jimma T et al. One thousand patients with primary myelofibrosis: the Mayo Clinic experience. Mayo Clin. Proc. 87(1), 25-33 (2012).

8. Elena C, Passamonti F, Rumi E et al. Red blood cell transfusion-dependency implies a poor survival in primary myelofibrosis irrespective of IPSS and DIPSS. Haematologica 96(1), 167-170 (2011).

9. Nicolosi M, Mudireddy M, Lasho TL et al. Sex and degree of severity influence the prognostic impact of anemia in primary myelofibrosis: analysis based on 1109 consecutive patients. Leukemia 1254-1258 (2018).

10. Passamonti F, Cervantes F, Vannucchi AM et al. A dynamic prognostic model to predict survival in primary myelofibrosis: a study by the IWG-MRT (International Working Group for Myeloproliferative Neoplasms Research and Treatment). Blood 115(9), 1703-1708 (2010).

-. This prognostic model highlights the importance of anemia in MF since moderate to severe anemia is scored twice as heavily as all other factors.

11. Gangat N, Caramazza D, Vaidya R et al. DIPSS plus: a refined dynamic international prognostic scoring system for primary myelofibrosis that incorporates prognostic information from karyotype, platelet count, and transfusion status. J. Clin. Oncol. 29(4), 392-397 (2011). 
12. Bartoszko J, Panzarella T, Lau A et al. Effect of red blood cell transfusion dependence on the natural history of myeloproliferative neoplasm-associated myelofibrosis. Clin. Lymphoma, Myeloma Leuk. 15(11), e151-e156 (2015).

13. Shander A, Cappellini MD, Goodnough LT. Iron overload and toxicity: the hidden risk of multiple blood transfusions. Vox Sang. 97(3), 185-197 (2009).

14. Harrison CN, Koschmieder S, Foltz L et al. The impact of myeloproliferative neoplasms (MPNs) on patient quality of life and productivity: results from the international MPN landmark survey. Ann. Hematol. 96(10), 1653-1665 (2017).

15. Scherber R, Dueck A, Johansson PL et al. The myeloproliferative neoplasm symptom assessment form (MPN-SAF): an international prospective validation trial. Blood 118(2), 401-409 (2011).

16. Tiribelli M, Palandri F, Sant'Antonio E, Breccia M, Bonifacio M. The role of allogeneic stem-cell transplant in myelofibrosis in the era of JAK inhibitors: a case-based review. Bone Marrow Transplant. 55(4), 708-716 (2020).

17. Pardanani A. JAK2 inhibitor therapy in myeloproliferative disorders: rationale, preclinical studies and ongoing clinical trials. Leukemia 22(1), 23-30 (2008).

18. Pardanani A, Gotlib JR, Jamieson C et al. Safety and efficacy of TG101348, a selective JAK2 inhibitor, in myelofibrosis. J. Clin. Oncol. 29(7), 789-796 (2011).

19. Cervantes F, Correa JG, Hernandez-Boluda JC. Alleviating anemia and thrombocytopenia in myelofibrosis patients. Expert Rev. Hematol. 9(5), 489-496 (2016).

20. Luo X, Xu Z, Li B et al. Thalidomide plus prednisone with or without danazol therapy in myelofibrosis: a retrospective analysis of incidence and durability of anemia response. Blood Cancer J. 8(1), 4-8 (2018).

21. Hernández-Boluda JC, Correa JG, García-Delgado R et al. Predictive factors for anemia response to erythropoiesis-stimulating agents in myelofibrosis. Eur. J. Haematol. 98(4), 407-414 (2017).

22. NCCN MPN Panel. Myeloproliferative neoplasms. (2020). https://www.nccn.org/professionals/physician_gls/pdf/mpn.pdf

23. Ganz T. Systemic iron homeostasis. Physiol. Rev. 93(4), 1721-1741 (2013).

24. Langdon JM, Yates SC, Femnou LK et al. Hepcidin-dependent and hepcidin-independent regulation of erythropoiesis in a mouse model of anemia of chronic inflammation. Am. J. Hematol. 89(5), 470-479 (2014).

25. Birgegard G, Samuelsson J, Ahlstrand E et al. Inflammatory functional iron deficiency common in myelofibrosis, contributes to anaemia and impairs quality of life. From the Nordic MPN Study Group. Eur. J. Haematol. 1-3 (2018).

26. Asshoff M, Warr M, Haschka D et al. Momelotinib inhibits ACVR1/ALK2, decreases hepcidin production, and ameliorates anemia of chronic disease in rodents. Blood 129(13), 1823-1830 (2017).

27. Oh ST, Talpaz M, Gerds AT et al. ACVR1/JAK1/JAK2 inhibitor momelotinib reverses transfusion dependency and suppresses hepcidin in myelofibrosis Phase 2 trial. Blood Adv. 4(18), (2020).

- Explores mechanisms underlying the activity of momelotinib (MMB) on MF-associated iron-restricted anemia, including its impact on serum hepcidin levels, and markers of iron storage and availability, erythropoiesis and inflammation.

28. Mesa R, Catalano J, Cervantes F. Dynamic and time-to-event analyses demonstrate marked reduction in transfusion requirements for janus kinase inhibitor-naïve myelofibrosis patients treated with momelotinib compared head to head with ruxolitinib. ASH 2019 Poster 1663 (2019).

http://filecache.investorroom.com/mr5ircnw_sierra/283/Sierra\%20MMB\%20ASH\%202019\%20Asbtract\%201663_Final.pdf

- This poster presents analyses of transfusion requirements during SIMPLIFY-1, demonstrating MMB's differentiated anemia benefit.

29. Gupta V, Miklós E, Hus M et al. Momelotinib dose-intensity is maintained in JAK inhibitor näive and previously JAK inhibitor treated intermediate/high risk myelofibrosis patients. EHA Poster EP1103 (2020). https://www.sierraoncology.com/wp-content/uploads/2020/06/EHA-2020_DI-Poster_Final.pdf

- This poster presents dose intensity of MMB and ruxolitinib during the SIMPLIFY studies; higher and sustained dose intensity was observed on momelotinib including for patients with ruxolitinib-induced hematological toxicity and/or progressive underlying loss of hematopoietic capacity.

30. Harrison C, Andreasson B, Cambier $\mathrm{N}$ et al. Long term safety of momelotinib in JAKi naïve and previously JAKi treated intermediate/high risk myelofibrosis patients. EHA Poster EP1113. (2020). https://www.sierraoncology.com/wp-content/uploads/2020/06/EHA-2020_Safety-Poster_Final.pdf

- This poster presents analyses of data from the SIMPLIFY studies, demonstrating the long-term safety of MMB.

31. Gwaltney C, Paty J, Kwitkowski VE et al. Development of a harmonized patient-reported outcome questionnaire to assess myelofibrosis symptoms in clinical trials. Leuk. Res. 59, 26-31 (2017).

-• Describes the questionnaire used to assess MF symptoms: the primary end point of this study.

32. Vannucchi AM, Barbui T, Cervantes F et al. Philadelphia chromosome-negative chronic myeloproliferative neoplasms: ESMO Clinical Practice Guidelines for diagnosis, treatment and follow-up. Ann. Oncol. 26(Suppl. 5), v85-v99 (2015).

33. Cervantes F. How I treat myelofibrosis. Blood 124(17), 2635-2642 (2014). 